\title{
Composite Stackelberg Strategy for Singularly Perturbed Bilinear Quadratic Systems
}

\author{
Ning BIN \\ School of Management, Guangdong University of Technology, Guangzhou 510520, China \\ E-mail:bn_gdut@163.com \\ Chengke ZHANG \\ School of Commerce \& Economics, Guangdong University of Technology, Guangzhou 510520, China \\ E-mail: chengke- $z @ 163 . c o m$ \\ Huainian ZHU \\ School of Management, Guangdong University of Technology, Guangzhou 510520, China \\ E-mail: huainian258@163.com \\ Zan MO \\ School of Management, Guangdong University of Technology, Guangzhou 510520, China \\ E-mail:mozan@126.com
}

\begin{abstract}
Based on singularly perturbed bilinear quadratic problems, this paper proposes to decompose the full-order system into two subsystems of a slow-time and fast-time scale. Utilizing the fixed point iterative algorithm to solve cross-coupled algebraic Riccati equations, equilibrium strategies of the two subsystems can be obtained, and further the composite strategy of the original full-order system. It was proved that such a composite strategy formed an $o(\varepsilon)$ (near) Stackelberg equilibrium, and a numerical result of the algorithm was presented in the end.
\end{abstract}

Keywords singularly perturbed; bilinear quadratic system; stackelberg equilibrium

\section{Introduction}

Dynamic game theory has been studied widely over the past decades, and the non-cooperative game theory of linear quadratic systems has been studied intensively in many papers. For example, Cruz. Jr et al. obtained the open-loop Stackelberg strategy in non-zero sum games ${ }^{[1]}$; in [2], Basar summarized the non-cooperative game theory in linear quadratic systems; in [3], Medanic developed necessary conditions for closed-loop Stackelberg strategies in linear quadratic problems and presented an algorithm for numerical solutions of two-level Stackelberg problems;

Received April 22, 2014, accepted September 1, 2014

Supported by the National Number Science Fund of China (71171061), 2014 Natural Science Fund of Guangdong Province (Non-cooperative Game Theory of Singularly Perturbed Markov System), Philosophy and Social Science "the Twelfth Five-Year" Plan Project of Guangdong Province (GD14YGL01), 2014 Guangzhou Philosophy and Social Science Project (14Q21) 
Mizukami investigated the linear quadratic closed-loop Stackelberg game for the descriptor system and constructed the incentive strategies in [4]. For singularly perturbed systems, in [5], Khalil and Kokotovic discussed the well-posedness of singularly perturbed Nash games and illustrated the impact of the feedback information available to players on the well-posedness of the game; Xu and Mizukami presented a unified approach to achieve the composite approximation of the full-order linear feedback saddle-point solution ${ }^{[6]}$; Mukaidani proposed a new algorithm for solving cross-coupled algebraic Riccati equations of singularly perturbed Nash games in [7], further applied the algorithm in obtaining the linear quadratic infinite horizon Nash game for general multiparameter singularly pertubed systems ${ }^{[8]}$, studied the computation of the linear closed-loop Stackelberg strategies with small singular perturbation parameter in [9], and investigated the linear closed-loop Stackelberg strategy of the singularly perturbed stochastic systems with state dependent noise ${ }^{[10]}$.

However, game theories of singularly perturbed bilinear systems are seldom discussed, while singularly perturbed bilinear systems are a quite proper and essential description tool in describing many practical systems such as neutron level control problem in a fission reactor, DC-motor, induction motor drives ${ }^{[11]}$, and in financial engineering problems, Black-Scholes Option Pricing Model, Aoki's two sector macroeconomic growth model, Chander and Tokao's non-linear input-output model can all be extended to singularly perturbed bilinear models in [12-15].

The structure of this paper is organized as follows. In Section 2, the problem of the differential Stackelberg equilibrium strategy for a singularly perturbed bilinear time-invariant system is presented. Sections 3 and 4 are concerned with the decomposition of the full-order system into two subsystems, and the composition strategy of the original full-order system. A simple numerical example is solved in Section 5. Section 6 contains the conclusion.

\section{Problem Statement}

Consider a time-invariant singularly perturbed bilinear system:

$$
\begin{aligned}
{\left[\begin{array}{l}
\dot{x}_{1}(t) \\
\varepsilon \dot{x}_{2}(t)
\end{array}\right]=} & {\left[\begin{array}{ll}
A_{11} & A_{12} \\
A_{21} & A_{22}
\end{array}\right]\left[\begin{array}{l}
x_{1}(t) \\
x_{2}(t)
\end{array}\right]+\left[\begin{array}{l}
B_{11} \\
B_{21}
\end{array}\right] u(t)+\left[\begin{array}{l}
B_{12} \\
B_{22}
\end{array}\right] v(t) } \\
& +\left\{\left[\begin{array}{l}
x_{1}(t) \\
x_{2}(t)
\end{array}\right]\left[\begin{array}{l}
M_{s} \\
M_{f}
\end{array}\right]\right\} u(t)+\left\{\left[\begin{array}{l}
x_{1}(t) \\
x_{2}(t)
\end{array}\right]\left[\begin{array}{l}
N_{s} \\
N_{f}
\end{array}\right]\right\} v(t)
\end{aligned}
$$

with initial condition

$$
\left[\begin{array}{l}
x_{1}(0) \\
x_{2}(0)
\end{array}\right]=\left[\begin{array}{l}
x_{10} \\
x_{20}
\end{array}\right]
$$

where $x_{1}(t) \in R^{n_{1}}, x_{2}(t) \in R^{n_{2}}$ are respectively slow and fast state variable, $x(t)=\left[x_{1}(t), x_{2}(t)\right]^{\mathrm{T}}$ $\in R^{n}$ are state vector with $n_{1}+n_{2}=n, u(t) \in R^{m}$ and $v(t) \in R^{l}$ are respectively the control inputs of Player 1 and Player 2, the small singular perturbation parameter $\varepsilon>0$ represents small time constants, inertias, masses, etc., and $A_{11}, A_{12}, A_{21}, A_{22}, B_{11}, B_{12}, B_{21}, B_{22}, M_{s}$, 
$M_{f}, N_{s}, N_{f}$ are constant matrices of appropriate dimensions, with

$$
\begin{aligned}
& \left\{\left[\begin{array}{l}
x_{1}(t) \\
x_{2}(t)
\end{array}\right]\left[\begin{array}{l}
M_{s} \\
M_{f}
\end{array}\right]\right\}=\sum_{j=1}^{n_{1}} x_{1 j}\left[\begin{array}{l}
M_{s j} \\
M_{f j}
\end{array}\right]+\sum_{j=n_{1}+1}^{n_{1}+n_{2}} x_{2 j}\left[\begin{array}{l}
M_{s j} \\
M_{f j}
\end{array}\right] \\
& \left\{\left[\begin{array}{l}
x_{1}(t) \\
x_{2}(t)
\end{array}\right]\left[\begin{array}{l}
N_{s} \\
N_{f}
\end{array}\right]\right\}=\sum_{j=1}^{n_{1}} x_{1 j}\left[\begin{array}{l}
N_{s j} \\
N_{f j}
\end{array}\right]+\sum_{j=n_{1}+1}^{n_{1}+n_{2}} x_{2 j}\left[\begin{array}{l}
N_{s j} \\
N_{f j}
\end{array}\right]
\end{aligned}
$$

The cost function for each player is defined by

$$
J_{i}(u, v)=\frac{1}{2} \int_{0}^{\infty}\left[x^{\mathrm{T}}(t) Q_{i} x(t)+u^{\mathrm{T}}(t) R_{i i} u(t)+v^{\mathrm{T}}(t) R_{i j} v(t)\right] \mathrm{d} t
$$

where

$$
R_{i i}>0, R_{i j}>0, i, j=1,2, i \neq j, \quad Q_{i}=\left[\begin{array}{cc}
Q_{i 11} & Q_{i 12} \\
Q_{i 12}^{\mathrm{T}} & Q_{i 22}
\end{array}\right]
$$

It is assumed that the decision-maker denoted by Player 1 is the leader, and Player 2 is the follower. Under the assumption that both players employ strategies $u:=u(x, t), v:=v(x, t)$, a strategy set $\left(u^{*}, v^{*}\right)$ is called a Stackelberg strategy if for any admissible strategy set $(u, v)$, the following conditions hold ${ }^{[10]}$.

$$
J_{1}\left(u^{*}, v^{*}\right) \leq J_{1}\left(u, v^{0}(u)\right), \quad \forall u \in R^{m}
$$

where

$$
J_{2}\left(u, v^{0}(u)\right)=\min _{v} J_{2}(u, v)
$$

and

$$
v^{*}=v^{0}\left(u^{*}\right)
$$

\section{Decomposition of Slow and Fast Systems}

Let

$$
\begin{aligned}
& {\left[\begin{array}{c}
\tilde{B}_{11}(x) \\
\tilde{B}_{21}(x)
\end{array}\right]=\left[\begin{array}{l}
B_{11} \\
B_{21}
\end{array}\right]+\left\{\left[\begin{array}{l}
x_{1} \\
x_{2}
\end{array}\right]\left[\begin{array}{l}
M_{s} \\
M_{f}
\end{array}\right]\right\}, \quad\left[\begin{array}{l}
\tilde{B}_{12}(x) \\
\tilde{B}_{22}(x)
\end{array}\right]=\left[\begin{array}{l}
B_{12} \\
B_{22}
\end{array}\right]+\left\{\left[\begin{array}{l}
x_{1} \\
x_{2}
\end{array}\right]\left[\begin{array}{l}
N_{s} \\
N_{f}
\end{array}\right]\right\}} \\
& \tilde{B}_{11}=\tilde{B}_{11}(x), \quad \tilde{B}_{21}=\tilde{B}_{21}(x), \quad \tilde{B}_{12}=\tilde{B}_{12}(x), \quad \tilde{B}_{22}=\tilde{B}_{22}(x)
\end{aligned}
$$

then (1) can be written as:

$$
\left\{\begin{array}{l}
\dot{x}_{1}=A_{11} x_{1}+A_{12} x_{2}+\tilde{B}_{11} u+\tilde{B}_{12} v \\
\varepsilon \dot{x}_{2}=A_{21} x_{1}+A_{22} x_{2}+\tilde{B}_{21} u+\tilde{B}_{22} v
\end{array}\right.
$$

Neglecting the fast modes is equivalent to assuming that they are infinitely fast, that is letting $\varepsilon=0$. Without the fast modes the system (5) reduces to

$$
\begin{aligned}
& \dot{x}_{1}=A_{11} x_{1}+A_{12} x_{2}+\tilde{B}_{11} u+\tilde{B}_{12} v \\
& 0=A_{21} x_{1}+A_{22} x_{2}+\tilde{B}_{21} u+\tilde{B}_{22} v
\end{aligned}
$$

Assuming that $A_{22}$ is nonsingular, we have

$$
\dot{x}_{1 s}=A_{0} x_{1 s}+\tilde{B}_{01} u_{s}+\tilde{B}_{02} v_{s}, \quad x_{1 s}=x_{10}
$$




$$
x_{2 s}=-A_{22}^{-1}\left(A_{21} x_{1 s}+\tilde{B}_{21} u_{s}+\tilde{B}_{22} v_{s}\right)
$$

where $A_{0}=A_{11}-A_{12} A_{22}^{-1} A_{21}, \tilde{B}_{01}=\tilde{B}_{11}-A_{12} A_{22}^{-1} \tilde{B}_{21}, \tilde{B}_{02}=\tilde{B}_{12}-A_{12} A_{22}^{-1} \tilde{B}_{22}$.

Then we can obtain the quadratic cost function for the slow subsystem

$$
\begin{gathered}
J_{i s}=\frac{1}{2} \int_{0}^{\infty}\left(x_{1 s}^{\mathrm{T}} Q_{i 0} x_{1 s}+2 x_{1 s}^{\mathrm{T}} D_{i 1} u_{s}+2 x_{1 s}^{\mathrm{T}} D_{i 2} v_{s}+2 u_{s}^{\mathrm{T}} D_{i 3} v_{s}\right. \\
\left.+u_{s}^{\mathrm{T}} R_{i 1 s} u_{s}+v_{s}^{\mathrm{T}} R_{i 2 s} v_{s}\right) \mathrm{d} t
\end{gathered}
$$

where $Q_{i 0}=Q_{i 11}+A_{21}^{\mathrm{T}} A_{22}^{-\mathrm{T}} Q_{i 22} A_{22}^{-1} A_{21}, D_{i 1}=A_{21}^{\mathrm{T}} A_{22}^{-\mathrm{T}} Q_{i 22} A_{22}^{-1} \tilde{B}_{21}, D_{i 2}=A_{21}^{\mathrm{T}} A_{22}^{-\mathrm{T}} Q_{i 22} A_{22}^{-1}$ $\tilde{B}_{22}, D_{i 3}=\tilde{B}_{21}^{\mathrm{T}} A_{22}^{-\mathrm{T}} Q_{i 22} A_{22}^{-1} \tilde{B}_{22}, R_{i 1 s}=R_{i 1}+\tilde{B}_{21}^{\mathrm{T}} A_{22}^{-\mathrm{T}} Q_{i 22} A_{22}^{-1} \tilde{B}_{21}, R_{i 2 s}=R_{i 2}+\tilde{B}_{22}^{\mathrm{T}} A_{22}^{-\mathrm{T}} Q_{i 22}$ $A_{22}^{-1} \tilde{B}_{22}$.

Theorem 1 Suppose that the following cross-coupled algebraic Riccati equations has solutions $p_{1 s}$ and $p_{2 s}$

$$
\begin{aligned}
& p_{1 s}\left(A-S_{1 s} p_{1 s}-S_{2 s} p_{2 s}\right)+\left(A-S_{1 s} p_{1 s}-S_{2 s} p_{2 s}\right)^{\mathrm{T}} p_{1 s}+p_{1 s} S_{1 s} p_{1 s}+Q_{1}=0 \\
& p_{2 s}\left(A-S_{1 s} p_{1 s}-S_{2 s} p_{2 s}\right)+\left(A-S_{1 s} p_{1 s}-S_{2 s} p_{2 s}\right)^{\mathrm{T}} p_{2 s}+p_{2 s} S_{2 s} p_{2 s}+Q_{2}=0
\end{aligned}
$$

where

$$
\begin{aligned}
& A=A_{0}+\tilde{B}_{01} T_{11}+\tilde{B}_{02} T_{21}, \quad S_{1 s}=\frac{1}{2}\left(\tilde{B}_{02} T_{22}-\tilde{B}_{01} T_{12}\right), \quad S_{2 s}=\frac{1}{2}\left(\tilde{B}_{01} T_{13}-\tilde{B}_{02} T_{23}\right) \\
& Q_{1}=Q_{10}+D_{11} T_{11}+D_{12} T_{21}, \quad Q_{2}=Q_{20}+D_{21} T_{11}+D_{22} T_{21}
\end{aligned}
$$

Then, the Stackelberg equilibrium solution $\left(u_{s}^{*}, v_{s}^{*}\right)$ of the slow subsystem can be given by

$$
\begin{aligned}
& u_{s}^{*}=\left(T_{11}+T_{12} p_{1 s}-T_{13} p_{2 s}\right) x_{1 s} \\
& v_{s}^{*}=\left(T_{21}-T_{22} p_{1 s}+T_{23} p_{2 s}\right) x_{1 s}
\end{aligned}
$$

Proof The Hamiltonian $H_{\text {is }}$ corresponding to the system (7) and performance index (8) is

$$
\begin{aligned}
H_{i s}= & \frac{1}{2}\left(x_{1 s}^{\mathrm{T}} Q_{i 0} x_{1 s}+2 x_{1 s}^{\mathrm{T}} D_{i 1} u_{s}+2 x_{1 s}^{\mathrm{T}} D_{i 2} v_{s}+2 u_{s}^{\mathrm{T}} D_{i 3} v_{s}+u_{s}^{\mathrm{T}} R_{i 1 s} u_{s}+v_{s}^{\mathrm{T}} R_{i 2 s} v_{s}\right) \\
& +\lambda_{i}^{\mathrm{T}}\left(A_{0} x_{1 s}+\tilde{B}_{01} u_{s}+\tilde{B}_{02} v_{s}\right)
\end{aligned}
$$

where $\lambda_{i} \in R^{n_{1} \times 1}$ is the Langrangian multiplier.

Given arbitrary $u_{s}$, the corresponding $v_{s}$ is obtained by minimizing $J_{2 s}$ with respect to $v_{s}$. Then, the optimal control is given by

$$
v_{s}=-R_{22 s}^{-1}\left(D_{22}^{\mathrm{T}} x_{1 s}+D_{23}^{\mathrm{T}} u_{s}+\tilde{B}_{02}^{\mathrm{T}} \lambda_{2}\right)
$$

Then the cost $J_{1 s}$ can be obtained, and we can further obtain

$$
\begin{aligned}
u_{s}= & \left(-R_{11 s}+2 D_{13} R_{22 s}^{-1} D_{23}^{\mathrm{T}}-D_{23} R_{22 s}^{-\mathrm{T}} R_{12 s} R_{22 s}^{-1} D_{23}^{\mathrm{T}}\right)^{-1} \\
& {\left[\begin{array}{l}
\left(D_{11}^{\mathrm{T}}-D_{23} R_{22 s}^{-\mathrm{T}} D_{12}^{\mathrm{T}}-D_{13} R_{22 s}^{-1} D_{22}^{\mathrm{T}}+D_{23} R_{22 s}^{-\mathrm{T}} R_{12 s} R_{22 s}^{-1} D_{22}^{\mathrm{T}}\right) x_{1 s} \\
+\left(\tilde{B}_{01}^{\mathrm{T}}-D_{23} R_{22 s}^{-\mathrm{T}} \tilde{B}_{02}^{\mathrm{T}}\right) \lambda_{1}+\left(D_{23} R_{22 s}^{-\mathrm{T}} R_{12 s}-D_{13}\right) R_{22 s}^{-1} \tilde{B}_{02}^{\mathrm{T}} \lambda_{2}
\end{array}\right] } \\
= & T_{11} x_{1 s}+T_{12} \lambda_{1}-T_{13} \lambda_{2}
\end{aligned}
$$


then

$$
\begin{aligned}
v_{s} & =-R_{22 s}^{-1}\left(D_{22}^{\mathrm{T}} x_{1 s}+D_{23}^{\mathrm{T}} u_{s}+\tilde{B}_{02}^{\mathrm{T}} \lambda_{2}\right) \\
& =-R_{22 s}^{-1} D_{22}^{\mathrm{T}} x_{1 s}-R_{22 s}^{-1} D_{23}^{\mathrm{T}} u_{s}-R_{22 s}^{-1} \tilde{B}_{02}^{\mathrm{T}} \lambda_{2} \\
& =-R_{22 s}^{-1} D_{22}^{\mathrm{T}} x_{1 s}-R_{22 s}^{-1} D_{23}^{\mathrm{T}}\left(T_{11} x_{1 s}+T_{12} \lambda_{1}-T_{13} \lambda_{2}\right)-R_{22 s}^{-1} \tilde{B}_{02}^{\mathrm{T}} \lambda_{2} \\
& =\left(-R_{22 s}^{-1} D_{22}^{\mathrm{T}}-R_{22 s}^{-1} D_{23}^{\mathrm{T}} T_{11}\right) x_{1 s}-R_{22 s}^{-1} D_{23}^{\mathrm{T}} T_{12} \lambda_{1}+\left(R_{22 s}^{-1} D_{23}^{\mathrm{T}} T_{13}-R_{22 s}^{-1} \tilde{B}_{02}^{\mathrm{T}}\right) \lambda_{2} \\
& =T_{21} x_{1 s}-T_{22} \lambda_{1}+T_{23} \lambda_{2}
\end{aligned}
$$

where

$$
\begin{aligned}
T_{11}= & \left(-R_{11 s}+2 D_{13} R_{22 s}^{-1} D_{23}^{\mathrm{T}}-D_{23} R_{22 s}^{-\mathrm{T}} R_{12 s} R_{22 s}^{-1} D_{23}^{\mathrm{T}}\right)^{-1} \\
& \left(D_{11}^{\mathrm{T}}-D_{23} R_{22 s}^{-\mathrm{T}} D_{12}^{\mathrm{T}}-D_{13} R_{22 s}^{-1} D_{22}^{\mathrm{T}}+D_{23} R_{22}^{-\mathrm{T}} R_{12 s} R_{22 s}^{-1} D_{22}^{\mathrm{T}}\right) \\
T_{12}= & \left(-R_{11 s}+2 D_{13} R_{22 s}^{-1} D_{23}^{\mathrm{T}}-D_{23} R_{22 s}^{-\mathrm{T}} R_{12 s} R_{22 s}^{-1} D_{23}^{\mathrm{T}}\right)^{-1}\left(\tilde{B}_{01}^{\mathrm{T}}-D_{23} R_{22 s}^{-\mathrm{T}} \tilde{B}_{02}^{\mathrm{T}}\right) \\
T_{13}= & \left(-R_{11 s}+2 D_{13} R_{22 s}^{-1} D_{23}^{\mathrm{T}}-D_{23} R_{22 s}^{-\mathrm{T}} R_{12 s} R_{22 s}^{-1} D_{23}^{\mathrm{T}}\right)^{-1}\left(D_{13}-D_{23} R_{22 s}^{-\mathrm{T}} R_{12 s}\right) R_{22 s}^{-1} \tilde{B}_{02}^{\mathrm{T}} \\
T_{21}= & -R_{22 s}^{-1} D_{22}^{\mathrm{T}}-R_{22 s}^{-1} D_{23}^{\mathrm{T}} T_{11} \\
T_{22}= & R_{22 s}^{-1} D_{23}^{\mathrm{T}} T_{12} \\
T_{23}= & R_{22 s}^{-1} D_{23}^{\mathrm{T}} T_{13}-R_{22 s}^{-1} \tilde{B}_{02}^{\mathrm{T}}
\end{aligned}
$$

For $-\dot{\lambda}_{1}=Q_{10} x_{1 s}+D_{11} u_{s}+D_{12} v_{s}+A_{0}^{\mathrm{T}} \lambda_{1},-\dot{\lambda}_{2}=Q_{20} x_{1 s}+D_{21} u_{s}+D_{22} v_{s}+A_{0}^{\mathrm{T}} \lambda_{2}$, letting $\lambda_{1}=p_{1 s} x_{1 s}$ and $\lambda_{2}=p_{2 s} x_{1 s}$, (9a) and (9b) can be derived respectively. This is the desired result.

In [8], Mukaidani proposed a fixed-point iterative algorithm for solving cross-coupled algebraic Riccati equations (9).

Assumption 1 The triplet $\left(A_{0}, \tilde{B}_{01}, \sqrt{Q_{1}}\right)$ and $\left(A_{0}, \tilde{B}_{02}, \sqrt{Q_{2}}\right)$ are stabilizable and detectable.

Under Assumption 1, the positive semidefinite solutions of cross-coupled algebraic Riccati equations (9) exist. It is obtained by performing the fixed-point algorithm:

$$
\begin{aligned}
& p_{1 s}^{(n+1)}\left(A-S_{1 s} p_{1 s}^{(n)}-S_{2 s} p_{2 s}^{(n)}\right)+\left(A-S_{1 s} p_{1 s}^{(n)}-S_{2 s} p_{2 s}^{(n)}\right)^{\mathrm{T}} p_{1 s}^{(n+1)}+Q_{1}+p_{1 s}^{(n) \mathrm{T}} S_{1 s} p_{1 s}^{(n)}=0 \\
& p_{2 s}^{(n+1)}\left(A-S_{1 s} p_{1 s}^{(n)}-S_{2 s} p_{2 s}^{(n)}\right)+\left(A-S_{1 s} p_{1 s}^{(n)}-S_{2} p_{2 s}^{(n)}\right)^{\mathrm{T}} p_{2 s}^{(n+1)}+Q_{2}+p_{2 s}^{(n) \mathrm{T}} S_{2 s} p_{2 s}^{(n)}=0 \\
& n=0,1,2, \cdots
\end{aligned}
$$

where $p_{1 s}^{(0)}, p_{2 s}^{(0)}$ are the solutions of the following algebraic Riccati equations:

$$
\begin{aligned}
& p_{1 s}^{(0)} A+A^{\mathrm{T}} p_{1 s}^{(0)}+Q_{1}-p_{1 s}^{(0) \mathrm{T}} S_{1 s} p_{1 s}^{(0)}=0 \\
& p_{2 s}^{(0)}\left(A-S_{1 s} p_{1 s}^{(0)}\right)+\left(A-S_{1 s} p_{1 s}^{(0)}\right)^{\mathrm{T}} p_{2 s}^{(0)}+Q_{2}-p_{2 s}^{(0) \mathrm{T}} S_{2 s} p_{2 s}^{(0)}=0
\end{aligned}
$$

The proof can be seen in [8].

In the fast subsystem, we assume that the slow variables are constant in the boundary layer. Redefining the fast variables $x_{2 f}=x_{2}-x_{2 s}$, and the fast controls $u_{f}=u-u_{s}, v_{f}=v-v_{s}$, the fast subsystem is formulated as:

$$
\dot{x}_{2 f}=\frac{1}{\varepsilon} A_{22} x_{2 f}+\frac{1}{\varepsilon} \tilde{B}_{21} u_{f}+\frac{1}{\varepsilon} \tilde{B}_{22} v_{f}, \quad x_{2 f}(0)=x_{20}-x_{2 s}(0)
$$


Then we can obtain the quadratic cost function for the fast subsystem

$$
J_{i f}=\frac{1}{2} \int_{0}^{\infty}\left(x_{2 f}^{\mathrm{T}} Q_{i 22} x_{2 f}+u_{f}^{\mathrm{T}} R_{i 1} u_{f}+v_{f}^{\mathrm{T}} R_{i 2} v_{f}\right) \mathrm{d} t
$$

Assumption 2 The triplet $\left(A_{22}, \tilde{B}_{21}, \sqrt{Q_{122}}\right)$ and $\left(A_{22}, \tilde{B}_{22}, \sqrt{Q_{222}}\right)$ are stabilizable and detectable.

Theorem 2 Under Assumption 2, suppose that the following cross-coupled algebraic Riccati equations has solutions $p_{1 f}$ and $p_{2 f}$

$$
\begin{aligned}
& p_{1 f}\left(A_{22}-S_{1 f} p_{1 f}-S_{2 f} p_{2 f}\right)+\left(A_{22}-S_{1 f} p_{1 f}-S_{2 f} p_{2 f}\right)^{\mathrm{T}} p_{1 f}+p_{1 f} S_{1 f} p_{1 f}+Q_{122}=0 \\
& p_{2 f}\left(A_{22}-S_{1 f} p_{1 f}-S_{2 f} p_{2 f}\right)+\left(A_{22}-S_{1 f} p_{1 f}-S_{2 f} p_{2 f}\right)^{\mathrm{T}} p_{2 f}+p_{2 f} S_{2 f} p_{2 f}+Q_{222}=0
\end{aligned}
$$

where $S_{1 f}=\frac{1}{2} \tilde{B}_{21} R_{11}^{-1} \tilde{B}_{21}^{\mathrm{T}}, S_{2 f}=\frac{1}{2} \tilde{B}_{22} R_{22}^{-1} \tilde{B}_{22}^{\mathrm{T}}$.

Then, the Stackelberg equilibrium solution $\left(u_{f}^{*}, v_{f}^{*}\right)$ of the fast subsystem can be given by

$$
\begin{aligned}
& u_{f}^{*}=-R_{11}^{-1} \tilde{B}_{21}^{\mathrm{T}} p_{1 f} x_{2 f} \\
& v_{f}^{*}=-R_{22}^{-1} \tilde{B}_{22}^{\mathrm{T}} p_{2 f} x_{2 f}
\end{aligned}
$$

Proof we can get the Stackelberg equilibrium solution $\left(u_{f}^{*}, v_{f}^{*}\right)$ of the fast subsystem

$$
v_{f}^{*}=-R_{22}^{-1} \tilde{B}_{22}^{\mathrm{T}} p_{2 f} x_{2 f}
$$

then

$$
\begin{aligned}
H_{1 f}= & \frac{1}{2}\left(x_{2 f}^{\mathrm{T}} Q_{122} x_{2 f}+u_{f}^{\mathrm{T}} R_{11} u_{f}+v_{f}^{\mathrm{T}} R_{12} v_{f}\right)+\lambda_{1 f}^{\mathrm{T}}\left(A_{22} x_{2 f}+\tilde{B}_{21} u_{f}+\tilde{B}_{22} v_{f}\right) \\
= & \frac{1}{2}\left(x_{2 f}^{\mathrm{T}} Q_{122} x_{2 f}+u_{f}^{\mathrm{T}} R_{11} u_{f}\right)+\lambda_{1 f}^{\mathrm{T}}\left(A_{22} x_{2 f}+\tilde{B}_{21} u_{f}\right) \\
& +\frac{1}{2} \lambda_{2 f}^{\mathrm{T}} \tilde{B}_{22} R_{22}^{-\mathrm{T}} R_{12} R_{22}^{-1} \tilde{B}_{22}^{\mathrm{T}} \lambda_{2 f}-\lambda_{1 f}^{\mathrm{T}} \tilde{B}_{22} R_{22}^{-1} \tilde{B}_{22}^{\mathrm{T}} \lambda_{2 f}
\end{aligned}
$$

where $\lambda_{i f} \in R^{n_{2} \times 1}$ is the Langrangian multiplier. Then

$$
u_{f}^{*}=-R_{11}^{-1} \tilde{B}_{21}^{\mathrm{T}} \lambda_{1 f}=-R_{11}^{-1} \tilde{B}_{21}^{\mathrm{T}} p_{1 f} x_{2 f}
$$

where $p_{1 f}, p_{2 f}$ satisfy the cross-coupled algebraic Riccati equations (17).

Similarly, under Assumption 2, the positive semidefinite solutions of cross-coupled algebraic Riccati equations (17) exist, and can be obtained by performing the fixed-point algorithm:

$$
\begin{aligned}
& p_{1 f}^{(n+1)}\left(A_{22}-S_{1 f} p_{1 f}^{(n)}-S_{2 f} p_{2 f}^{(n)}\right)+\left(A_{22}-S_{1 f} p_{1 f}^{(n)}\right. \\
& \left.\quad-S_{2 f} p_{2 f}^{(n)}\right)^{\mathrm{T}} p_{1 f}^{(n+1)}+Q_{122}+p_{1 f}^{(n) \mathrm{T}} S_{1 f} p_{1 f}^{(n)}=0 \\
& p_{2 f}^{(n+1)}\left(A_{22}-S_{1 f} p_{1 s}^{(n)}-S_{2 f} p_{2 f}^{(n)}\right)+\left(A_{22}-S_{1 f} p_{1 s}^{(n)}\right. \\
& \left.\quad-S_{2 f} p_{2 f}^{(n)}\right)^{\mathrm{T}} p_{2 f}^{(n+1)}+Q_{222}+p_{2 f}^{(n) \mathrm{T}} S_{2 f} p_{2 f}^{(n)}=0 \\
& n=0,1,2,3 \ldots
\end{aligned}
$$

where $p_{1 f}^{(0)}, p_{2 f}^{(0)}$ are the solutions of the following algebraic Riccati equations:

$$
\begin{aligned}
& p_{1 f}^{(0)} A_{22}+A_{22}^{\mathrm{T}} p_{1 f}^{(0)}+Q_{122}-p_{1 f}^{(0) \mathrm{T}} S_{1 f} p_{1 f}^{(0)}=0 \\
& p_{2 f}^{(0)}\left(A_{22}-S_{1 f} p_{1 f}^{(0)}\right)+\left(A_{22}-S_{1 f} p_{1 f}^{(0)}\right)^{\mathrm{T}} p_{2 f}^{(0)}+Q_{222}-p_{2 f}^{(0) \mathrm{T}} S_{2 f} p_{2 f}^{(0)}=0
\end{aligned}
$$




\section{Composite Strategy}

The composite Stackelberg strategy pair of the full-order singularly perturbed system (1) is constructed as follows ${ }^{[16]}$ :

$$
\begin{aligned}
& u_{c}=u_{s}^{*}+u_{f}^{*}=\left(T_{11}+T_{12} p_{1 s}-T_{13} p_{2 s}\right) x_{1 s}-R_{11}^{-1} \tilde{B}_{21}^{\mathrm{T}} p_{1 f} x_{2 f} \\
& v_{c}=v_{s}^{*}+v_{f}^{*}=\left(T_{21}-T_{22} p_{1 s}+T_{23} p_{2 s}\right) x_{1 s}-R_{22}^{-1} \tilde{B}_{22}^{\mathrm{T}} p_{2 f} x_{2 f}
\end{aligned}
$$

With $x_{1}$ replacing $x_{1 s}, x_{2}$ replacing $x_{2 s}+x_{2 f}$, for $x_{2 s}=-A_{22}^{-1}\left(A_{21} x_{1 s}+\tilde{B}_{21} u_{s}+\tilde{B}_{22} v_{s}\right)$, we obtain

$$
\begin{aligned}
& u_{c}=G_{1} x_{1}+G_{2} x_{2} \\
& v_{c}=G_{3} x_{1}+G_{4} x_{2}
\end{aligned}
$$

where

$$
\begin{aligned}
G_{1}= & \left(T_{11}+T_{12} p_{1 s}-T_{13} p_{2 s}\right)-R_{11}^{-1} \tilde{B}_{21}^{\mathrm{T}} p_{1 f} A_{22}^{-1}\left[A_{21}+\tilde{B}_{21}\left(T_{11}+T_{12} p_{1 s}-T_{13} p_{2 s}\right)\right. \\
& \left.+\tilde{B}_{22}\left(T_{21}-T_{22} p_{1 s}+T_{23} p_{2 s}\right)\right] \\
G_{2}= & -R_{11}^{-1} \tilde{B}_{21}^{\mathrm{T}} p_{1 f} \\
G_{3}= & \left(T_{21}-T_{22} p_{1 s}+T_{23} p_{2 s}\right)-R_{22}^{-1} \tilde{B}_{22}^{\mathrm{T}} p_{2 f} A_{22}^{-1}\left[A_{21}+\tilde{B}_{21}\left(T_{11}+T_{12} p_{1 s}-T_{13} p_{2 s}\right)\right. \\
& \left.+\tilde{B}_{22}\left(T_{21}-T_{22} p_{1 s}+T_{23} p_{2 s}\right)\right] \\
G_{4}= & -R_{22}^{-1} \tilde{B}_{22}^{\mathrm{T}} p_{2 f}
\end{aligned}
$$

Theorem 3 The composite strategy pair constitutes an o( $(\varepsilon)$ (near) Stackelberg equilibrium of the full-order game, that is,

$$
\begin{aligned}
& x_{1}(t)=x_{1 s}(t)+o(\varepsilon) \\
& x_{2}(t)=-A_{22}^{-1}\left(A_{21}+G_{0}\right) x_{1 s}(t)+x_{2 f}(t)+o(\varepsilon) \\
& u^{*}(t)=u_{c}(t)+o(\varepsilon) \\
& v^{*}(t)=v_{c}(t)+o(\varepsilon)
\end{aligned}
$$

Proof The feedback system (5) can be written as

$$
\left[\begin{array}{c}
\dot{x}_{1} \\
\varepsilon \dot{x}_{2}
\end{array}\right]=\left[\begin{array}{cc}
A_{11}+\tilde{B}_{11} G_{1}+\tilde{B}_{12} G_{3} & A_{12}+\tilde{B}_{11} G_{2}+\tilde{B}_{12} G_{4} \\
A_{21}+\tilde{B}_{21} G_{1}+\tilde{B}_{22} G_{3} & A_{22}+\tilde{B}_{21} G_{2}+\tilde{B}_{22} G_{4}
\end{array}\right]\left[\begin{array}{c}
x_{1} \\
x_{2}
\end{array}\right]
$$

Introducing the Chang transformation and its inverse

$$
T=\left[\begin{array}{cc}
I_{1}-\varepsilon H L & -\varepsilon H \\
L & I_{2}
\end{array}\right], \quad T^{-1}=\left[\begin{array}{cc}
I_{1} & \varepsilon H \\
-L & I_{2}-\varepsilon H L
\end{array}\right]
$$

while the transformation equations are given by

$$
\left\{\begin{array}{l}
\varepsilon L A_{11}+A_{21}-\left(\varepsilon L A_{12}+A_{22}\right) L=0 \\
A_{12}+\varepsilon\left(A_{11}-A_{12} L\right) H-H\left(\varepsilon L A_{12}+A_{22}\right)=0
\end{array}\right.
$$

we get

$$
T S T^{-1}=\left[\begin{array}{cc}
S_{1} & 0 \\
0 & S_{2}
\end{array}\right]
$$


where $S$ is the system matrix of (24),

$$
\begin{aligned}
S_{1} & =\left(A_{11}+\tilde{B}_{11} G_{1}+\tilde{B}_{12} G_{3}\right)-\left(A_{12}+\tilde{B}_{11} G_{2}+\tilde{B}_{12} G_{4}\right) L \\
& -\varepsilon H\left(A_{21}+\tilde{B}_{21} G_{1}+\tilde{B}_{22} G_{3}\right)-\varepsilon H L\left(A_{11}+\tilde{B}_{11} G_{1}+\tilde{B}_{12} G_{3}\right) \\
& +\varepsilon H L\left(A_{12}+\tilde{B}_{11} G_{2}+\tilde{B}_{12} G_{4}\right) L+\varepsilon H\left(A_{22}+\tilde{B}_{21} G_{2}+\tilde{B}_{22} G_{4}\right) L \\
S_{2} & =\left(A_{22}+\tilde{B}_{21} G_{2}+\tilde{B}_{22} G_{4}\right)+L\left(A_{12}+\tilde{B}_{11} G_{2}+\tilde{B}_{12} G_{4}\right) \\
& +L\left(A_{11}+\tilde{B}_{11} G_{1}+\tilde{B}_{12} G_{3}\right) \varepsilon H+\left(A_{21}+\tilde{B}_{21} G_{1}+\tilde{B}_{22} G_{3}\right) \varepsilon H \\
& -L\left(A_{12}+\tilde{B}_{11} G_{2}+\tilde{B}_{12} G_{4}\right) \varepsilon H L-\left(A_{22}+\tilde{B}_{21} G_{2}+\tilde{B}_{22} G_{4}\right) \varepsilon H L
\end{aligned}
$$

If $\left(A_{22}+\tilde{B}_{21} G_{2}+\tilde{B}_{22} G_{4}\right)+L\left(A_{12}+\tilde{B}_{11} G_{2}+\tilde{B}_{12} G_{4}\right)$ is stable, the solution of (24) is approximated for all finite $t \geq 0$ by

$$
\begin{aligned}
x_{1}(t)= & \exp \left[\left(A_{11}+\tilde{B}_{11} G_{1}+\tilde{B}_{12} G_{3}-A_{12} L-\tilde{B}_{11} G_{2} L-\tilde{B}_{12} G_{4} L\right) t\right] x_{1 s}(0)+o(\varepsilon) \\
x_{2}(t)= & -A_{22}^{-1}\left(A_{21}+G_{0}\right) \exp \left[\left(A_{11}+\tilde{B}_{11} G_{1}+\tilde{B}_{12} G_{3}-A_{12} L\right.\right. \\
& \left.\left.-\tilde{B}_{11} G_{2} L-\tilde{B}_{12} G_{4} L\right) t\right] x_{1 s}(0)+\exp \left[\left(A_{22}+\tilde{B}_{21} G_{2}+\tilde{B}_{22} G_{4}+L A_{12}\right.\right. \\
& \left.\left.+L \tilde{B}_{11} G_{2}+L \tilde{B}_{12} G_{4}\right) t / \varepsilon\right] x_{2 f}(0)+o(\varepsilon)
\end{aligned}
$$

where $x_{1 s}(0), x_{2 f}(0)$ are given by $(7 \mathrm{a})$, (15). If in addition $\left(A_{11}+\tilde{B}_{11} G_{1}+\tilde{B}_{12} G_{3}\right)-\left(A_{12}+\right.$ $\left.\tilde{B}_{11} G_{2}+\tilde{B}_{12} G_{4}\right) L$ is also stable, (28) holds for all $t \in[0, \infty)$. Then (23) follows directly from (28), (7) and (15).

\section{A Numerical Example}

In order to demonstrate the efficiency of the proposed decomposition method, we have run a simple numerical example. All matrices are chosen randomly, which are given by

$$
\begin{aligned}
& A_{11}=\left[\begin{array}{ll}
0 & 0.4 \\
0 & 0
\end{array}\right], \quad A_{12}=\left[\begin{array}{rr}
0 & 0 \\
0.345 & 0
\end{array}\right], \quad A_{21}=\left[\begin{array}{cc}
0 & -0.524 \\
0 & 0
\end{array}\right], \quad A_{22}=\left[\begin{array}{cc}
-0.465 & 0.262 \\
0 & -1
\end{array}\right], \\
& B_{11}=\left[\begin{array}{l}
0 \\
0
\end{array}\right], \quad B_{12}=\left[\begin{array}{l}
0 \\
0
\end{array}\right], \quad B_{21}=\left[\begin{array}{l}
0 \\
1
\end{array}\right], \quad B_{22}=\left[\begin{array}{c}
0.2 \\
1
\end{array}\right], \\
& M_{1}=N_{1}=\left[\begin{array}{l}
1 \\
0 \\
0 \\
0
\end{array}\right], \quad M_{2}=N_{2}=\left[\begin{array}{l}
0 \\
1 \\
0 \\
0
\end{array}\right], \quad M_{3}=N_{3}=\left[\begin{array}{l}
0 \\
0 \\
1 \\
0
\end{array}\right], \quad M_{4}=N_{4}=\left[\begin{array}{l}
0 \\
0 \\
0 \\
1
\end{array}\right]
\end{aligned}
$$

and a quadratic cost function

$$
\begin{aligned}
& J_{1}(u, v)=\frac{1}{2} \int_{0}^{\infty}\left(x^{\mathrm{T}} Q_{1} x+u^{2}+2 v^{2}\right) \mathrm{d} t \\
& J_{2}(u, v)=\frac{1}{2} \int_{0}^{\infty}\left(x^{\mathrm{T}} Q_{2} x+2 u^{2}+v^{2}\right) \mathrm{d} t
\end{aligned}
$$

where

$$
Q_{1}=\operatorname{diag}\{1,0,1,0\}, \quad Q_{2}=\operatorname{diag}\{1,0,1,0\}, \quad x_{10}=x_{20}=\left[\begin{array}{l}
1 \\
1
\end{array}\right]
$$


The simulation result is presented in Figure 1.

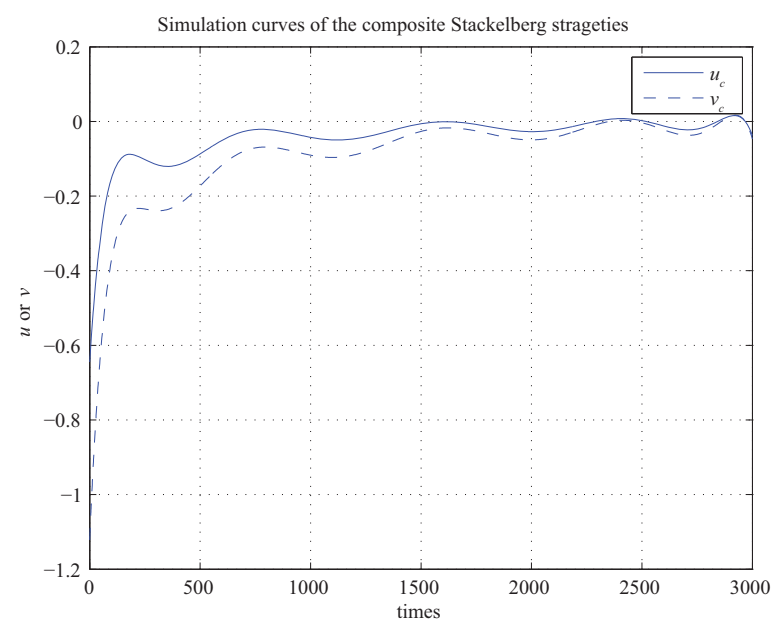

Figure 1 Simulation curves of the composite Stackelberg strategy $\left(u_{c}, v_{c}\right)$

\section{Conclusions}

Many real systems possess the structure of the singularly perturbed bilinear control systems such as motor drives, robust control, multi-sector input-output analysis and option pricing. In this paper, we have studied the Stackelberg games for singularly perturbed bilinear systems. And we propose to decompose the full-order system into two subsystems of a slow-time and fast-time scale. Utilizing the fixed point iterative algorithm to solve cross-coupled algebraic Riccati equations, equilibrium strategies of the two subsystems can be obtained, and further the composite strategy of the original full-order system. It has been proved that such a composite strategy formed an $o(\varepsilon)$ (near) Stackelberg equilibrium, and a numerical example in the end has demonstrated the efficiency of the algorithm. The conclusion obtained in this paper could be applied to deal with many practical industry engineering and financial engineering problems.

\section{References}

[1] Simaan M, Cruz Jr J B. On the Stackelberg strategy in non-zero sum games. Journal of Optimization Theory and Applications, 1973, 11(5): 533-555.

[2] Basar T, Olsder G T. Dynamic non-cooperative game theory. Academic Press, New York, 1991.

[3] Medanic J. Closed-loop Stackelberg strategies in linear-quadratic problems. IEEE Transactions on Automatic Control, 1978, 23(4): 632-637.

[4] Mizukami K, Xu H. Closed-loop stackelberg strategies for linear-quadratic descriptor systems. Journal of Optimization Theory and Applications, 1992, 74: 151-170.

[5] Khalil H K, Kokotovic P V. Feedback and well-posedness of singularly perturbed Nash games. IEEE Transactions on Automatic Control, 1979, 24(5): 699-708.

[6] Xu H, Mizukami K. Infinite-horizon differential games of singularly perturbed systems: A unified approach. Automatica, 1997, 33(2): 273-276.

[7] Mukaidani H, Xu H, Mizukami K. A new algorithm for solving cross-coupled algebraic Riccati equations of singularly perturbed Nash games. Proceedings of the 39th IEEE Conference on Decision and Control, Sydney, Australia, 2000: 3648-3653.

[8] Mukaidani H. A computational efficient numerical algorithm for solving cross-coupled algebraic Riccati 
equation and its application to multimodeling systems. Proceedings of the 2006 American Control Conference, Minneapolis, Minnesota, USA, 2006: 725-730.

[9] Mukaidani H. Efficient numerical procedures for solving closed-loop Stackelberg strategies with small singular perturbation parameter. Applied Mathematics and Computation, 2007, 188: 1173-1183.

[10] Mukaidani H, Unno M, Yamamoto T. Stackelberg strategies for singularly perturbed stochastic systems. Proceedings of the 2013 European Control Conference, Zurich, Switzerland, 2013: 730-735.

[11] Aganovic Z, Gajic Z. Linear optimal control of bilinear systems with applications to singularly perturbations and weak coupling. Springer-Verlag, London, 1995.

[12] Mceneaney W. A robust control framework for option pricing. Mathematics of Operations Research, 1997, 22(1): 203-221.

[13] Aoki M. Some examples of dynamic bilinear models in economics. Lecture Notes in Economics and Mathematical Systems, Springer-Verlag, Berlin Heidelberg, 1975: 163-169.

[14] Chander P. The nonlinear input output model. Journal of Economic Theory, 1983, 30: 219-229.

[15] Tokao F. Nonlinear Leontief model in abstract spaces. Journal of Mathematical Economics, 1986, 15: 151156.

[16] Kim B S, Lim M T. Composite control for singularly perturbed bilinear systems via successive Galerkin approximation. IEEE Proceedings of Control Theory, 2003, 150(5): 483-488. 